\title{
A New Framework for Designing E-Government Procurement in China Based on Ontology and Business Component*
}

\author{
Nan Lin ${ }^{1}$, Daifeng Li ${ }^{2}$, Tianxi Dong ${ }^{3}$, Zheng Qin ${ }^{2}$ \\ ${ }^{1}$ School of International Business Administration, Shanghai University of Finance \& Economic, Shanghai, China; ${ }^{2}$ School of Infor- \\ mation Management \& Engineering, Shanghai University of Finance \& Economic, Shanghai, China; ${ }^{3}$ Rawls College of Business, \\ Texas Tech University, TX, USA. \\ Email: linn@mail.shufe.edu.cn, *1df3824@yahoo.com.cn, dongtianxi@hotmail.com, qinzheng@mail.shufe.edu.cn
}

Received April 26 ${ }^{\text {th }}, 2010$; revised June $5^{\text {th }}, 2010$; accepted July $8^{\text {th }}, 2010$.

\begin{abstract}
Electronic government (e-government) procurement is one of the most important activities in China. The paper consists of three parts. First, the paper introduces current situation of China's e-government procurement system which includes the overall technical level, application level, and the existing problems. Based on the problem brought forward from the first part, the paper considers that a better solution is to apply business component theory and business component framework in the construction of e-government procurement, as it can solve the problems that block the development of e-government procurement in a more convenient way. The paper constructs the Business Component (BC) framework for the e-government procurement, analyzes the superiority of $B C$ framework and describes a methodology for the application of BCs in e-government procurement. The paper utilizes semantic model for workflow by using ontology modeling tool Protégé, uses ontology model database to store and manage workflow model, and builds a permission-based and user-involved workflow. At last, the paper takes public bidding, a main e-procurement method in China as an example and uses Appfuse and Osworkflow to prove the validity of the framework and methodology.
\end{abstract}

Keywords: Business Component, E-Government Procurement, Appfuse, Osworkflow, Ontology, Protégé

\section{Introduction}

E-government procurement is gaining more attention in the research field recently. Government administration is a kind of knowledge-intensive process from the view of service type $[1,2]$. Participants in the process must possess some professional knowledge of different industry fields, which makes the process more flexible and changeable. These features are more remarkable in the e-government procurement.

In China, government procurement refers to state organs, institutions and organizations at all levels that use fiscal funds to centrally purchase goods, works and services within a procurement directory or procurement standards noted above. The process of procurement is limited in a strict way. The most complex, important and representative method is public bidding (a main e-procurement method in China). It helps the govern-

*This paper is a thorough extension of a paper of ICDS 2008 (Li, et al., 2008). ment to obtain better working equipment or service in a relative low price, improve the working efficiency and reduce financial expense [3]. In China, The standard structure of government procurement can be seen in Figure 1.

In general, a municipal level of government procure-

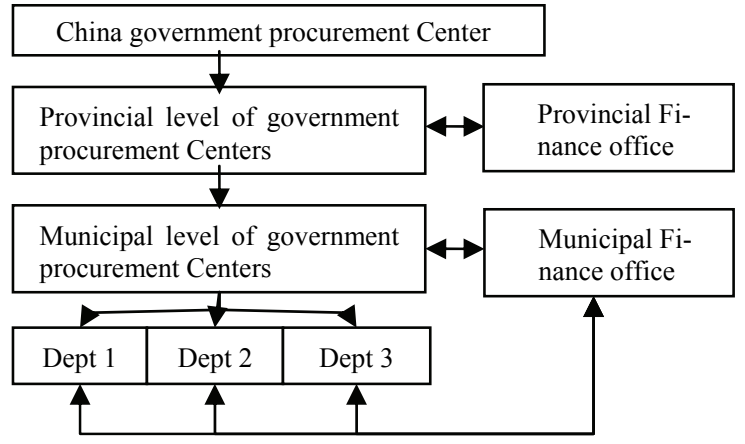

Figure 1. Structure of government procurement in China. 
ment Center usually contains three departments. As is seen in Figure 1, Dept1 represents the marketing office, whose main function is responsible for commodity procurement; Dept 2 represents the procurement office, whi$\mathrm{ch}$ is responsible for procurement of large-scale projects. Dept3 represents the integrated office, which is mainly responsible for coordinating the relationship among other departments.

Government procurement has gradually become more standardized and large-scale in recent years. The scale of government procurement is more than 590 billion, up $27 \%$, and capital saving rate of $11.3 \%$ [4]. In 2006, China issued the standards of e-government procurement, including standards of architecture, security, and selection of software components, plug-in software, and the functional requirements which should be achieved.

Different with other government office automation (OA) system and e-government citizens-oriented service system, e-government procurement is a special system that is a process of transaction between governments and enterprises. The e-government procurement has its unique characteristics.

According to the analysis above, the system of government procurement requires high level of flexibility, agility and adaptability because it is knowledge-intensive and its process should be changed frequently. The paper considers that Business Component $(\mathrm{BC})$ theory and its framework can fit the demands of e-government procurement commendably.

Definition: Business component (BC) represents a kind of software implementation of an "autonomous" business concept or business process. It is composed of all software components necessary to express, implement, and deploy a business component as an autonomous, reusable element of an information system [5].

BC theory's main idea is "to build on-demand". In this paper, to apply BC framework and ontology theory in e-government procurement, we should have an indepth understanding on that area and a mature method to build concept model. We use FODA (Feature-Oriented Domain Analysis) [6] to make analysis on the function and process of e-government procurement and use entity-append [7] approach to design BCs.

The structure of the paper is as follows: in Subsection 2.1, we use FODA to analyze the function and logic process of government procurement, build domain $\mathrm{BC}$ to identify index system and utilize cluster algorithm [8-11] to abstract and design BCs that can be reused in e-government procurement. In this section, the paper summarizes the superiority of each business component framework and designs a unique framework for e-government procurement by consulting Component-Based Software Development (CBSD) [12] for
BCs to deploy. In Subsection 2.2, the paper introduces the structure of a typical BC. In Subsection 2.3, the paper discusses how to use J2EE light weight architecture to develop the system under BC framework. In Subsection 2.4, a user participation workflow based on ontology is introduced to enhance the flexibility of the framework. In Section 3, an instance is introduced to describe how to use the framework to develop public bidding (a main procurement process approach) to validate feasibility and superiority of the system.

\section{Business Component Framework Based on Light Weight J2EE Architecture}

\subsection{Business Component Framework}

In this paper, based on traditional BC framework, we make some improvement and innovation on it. The research on BC framework is mature, but there is no related research on the application of $\mathrm{BC}$ framework in government procurement in China, so the main purpose of this paper is to analyze the domain characteristics of government procurement in China and build up a domain BC framework that can support government procurement in China more efficiently. The main innovation is listed below:

1) Use FODA to analyze the particular domain of government procurement in China.

2) Consider the advantages of several main $\mathrm{BC}$ framework $[6,8,9,13,14]$ that can be used in the government procurement, reconstruct them to meet the requirement of government procurement and research on how to integrate them into J2EE light weight architecture.

3) Research on how to make use of those advantages to construct $\mathrm{BC}$ framework, abstract and design BCs that is based on government procurement in China.

4) Adopt several evaluation approaches to estimate the effect of the BC framework that is built for government procurement.

First, we make domain classification from a strategic level. We consider that the research object cannot be limited to existing procurement but we also need some forward-looking about the development of government procurement. The organization structure should be meaningful in strategic level, and thus we adopt a coordination mechanism among decision-making, implementation, and supervision [4]. We can use that mechanism to guide the construct of government procurement system.

According to the analysis above, the domain can be divided into four main categories: decision categories, implementation categories, supervision categories and finance categories. The main function of decision categories is to decide the evaluation index system of bidding for a project, use qualitative and quantitative methods to 
analyze each bidder and judge which bidder can be successful in the bidding. Implementation categories implement the decision made by decision-makers, mainly including examining, approving and generating basic index table, product or project information table, user requirement table and etc. Finance categories add finance information to the basic table in accordance with the information the table has offered.

Each category has a BC database which contains a collection of standard reusable BCs, and the BC database consults Jun Ginbayashi and Rieko Yamamoto's Component AA mechanism [13], builds a detailed data model, and maps the model to the object. By using that approach and entity-append mechanism, we divide BCs into entity components (ECs), process components (PCs), abstract domain business components (ADBCs), business-level core business components (BCBCs), abstract core business components (ACBCs), customer core business components (CCBCs).

ADBCs are the proper characteristics in e-government procurement, which encapsulates the process and functions that is regulated by law and cannot be modified. They build the system frame to integrate other BCs. PCs is the set of meta-process which can describe concrete section of business process, while ECs is the set of meta-function which can realize some function of the system. ACBCs and BCBCs is the organic combination of ECs and PCs. The difference is that ACBCs is a kind of general $\mathrm{BC}$, that means the attributes and functions it provides are common and can be reused not only in e-government procurement domain but also in other domain such as OA or distribution system, and inherit $[15,16]$ mechanism is used to realize that general $\mathrm{BC}$, but $\mathrm{BCBC}$ considers the reusable in the same domain, it abstracts the common properties of several similar processes in the same domain and encapsulates those properties into a $\mathrm{BCBC}$, it has several parameters, and it can switch to different process by re-configuring the parameters. By using IoC $[16,17]$ mechanism, the parameters can directly point out which template model should be applied. So when changes happen, we just modify the parameters in service layer, then the rest work can be done automatically by using IoC mechanism. CCBMs emphasize the requirement of customers and design the business components that can satisfy the specific demands of customers.

Papers [9-11] introduce the clustering methods to identify and design business component from the set of metaprocess and meta-function. According to the study of those articles, we summarize their principles and use their methods to cluster meta-function and meta-process:

What's more, each category contains four modules in the $\mathrm{BC}$ framework, which are mathematic base, knowl- edge base, organization management module and template module. Mathematic base provides many kinds of mathematic tools and decision algorithms to support data analysis and make decision. Knowledge base provides an expert system for users to realize knowledge management and sharing. Organization management module defines the roles and authority of the system, and use user authentication mechanism to realize the dynamic banding between user and role [17]. Template module considers that one $\mathrm{BC}$ may choose different actions when confronting different situations, so it builds many templates to deal with changes. The structure of the framework can be seen in Figure 3.

The framework is divided into four layers that are user interface (UI) layer, business process layer, service layer, data operation layer and data layer. UI layer contains User Interface Business Components (UIBCs) which can make various UI interfaces for users to communicate with computers and many kinds of tables. Business process layer describes the logic of real business process and builds model for it by using Osworkflow (a kind of open source workflow software which is developed by Java). It divides the whole process into several stages, each stage is further divided into several activities, and each activity is a concrete task that is assigned to certain person. It needs BCs from service layer to implement response work. The main management tools of service layer are Application Context Affairs. All BCs should register in service layer, provide their addresses, functions and interfaces to Application Context Affairs so that it can find and use them, which are included in $\mathrm{BC}$ management.

\subsection{Structure of Business Component}

According to Subsection 2.1, BCs are divided into several types including ADBCs, BCBCs, ACBCs, CCBCs, ECs and PCs. Each business components should contain at least one of the two parts: PCs and ECs. Take ADBCs as an example, whose process is composed of several groups of meta-processes and entity is also consisted of several groups of meta-functions [11-13]. There is an organic link between meta-process and meta-function. We consider the meta-process and related meta-function as an individual object, and the object can exhibit typical structure of Business Component. The construct of the objects can be seen in Figure 2.

The structure has two layers that are meta-process layer and meta-function layer. Meta-process layer uses business interface to obtain the external information and service requirement, then sends them to the business proxy, and business proxy uses IoC mechanism [16-19] to select specify business service to realize service requirement. 


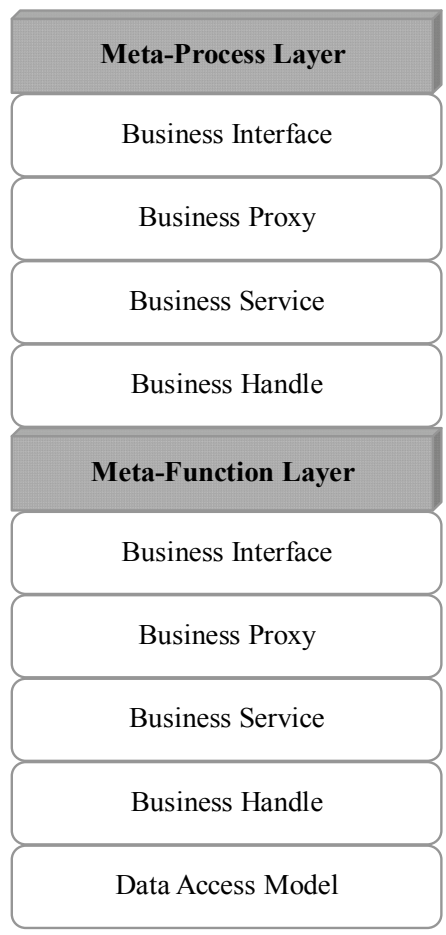

Figure 2. Structure of object.

The services in meta-process layer should be supported by the meta-function layer, which provides functions to realize the services that are brought by metaprocess layer in the same way as the meta-process does, but the different is that meta-function layer need to handle the database. As shown in Figure 3, the ADBCs need to encapsulate the operation of database too.

In this paper, we adopt Hibernate to obtain data object, and deal with the database just like invoking a general programming but does not need to insert into specify SQL language.

\subsection{Application of Appfuse and Osworkflow}

The main tools applied in the component based framework are EJB, web service and COM. Considering the feature of e-government procurement that is introduced in Section 1, we decide to choose a more agile architecture which integrates much technical superiority, possess nicer architecture support that can make flexible configuration. Light weight J2EE architecture is a good option, and Appfuse is one of the most excellent products in light weight J2EE architecture. It integrates the main software design frameworks of java such as Struts, Spring and Hibernate so we can use their respective specialties to construct three layers architecture that is inherited and abstracted from business component based methodology. It uses templates and configuration files to support fast development. Strong configuration function is a main characteristic in business component based framework that is described in literature [18].

In this paper, considering frameworks' respective advantages, we use Osworkflow to design the process of e-government procurement and utilize Appfuse to build the business component based architecture and design the management mechanism of business components [20,21]. We use Osworkflow to design the meta-process models and assemble them to form an integrate e-government procurement process, use JSP and Servlet to design UI and transfer information to Osworkflow, use Spring to implement and manage business components, and use Hibernate to implement database CRUD operation.

\subsection{User Participation Workflow Model Based on Ontology}

The e-government procurement is an interaction process among different enterprises, government departments and organizations, which involves different auditing processes, The processes are different and changed frequently, thus, the functions of the roles are also changed, so does the structures of the organizations. It is hard to define all the process and obtain the meta-process by usual method. Therefore, it is difficult to satisfy user's requirement. For example, in the past, government used inquiry approach for purchasing products and projects, the total value of which are less than 50 million Yuan. Now, in order to improve work efficiency, the government adopts fixed procurement method for different products. It is very difficult for us to update existing system to satisfy the changes. Redevelopment is considered as a waste of money and time and we also cannot be certain that the new system could be competent in the future.

So one method to solve that problem is to give users the capability to define their own workflow and organizations based on the models we provide. Of course, users can only define and modify a certain part of the workflow according to their authorities.

One of the main problems is how to use a more convenient way to help the users (without computer science background) to describe their ideas, and let computers know and implement them. The paper brings forward a solution, and applies that solution in the construction of e-government procurement primarily. We use ontology to build up a domain database. Ontology is a kind of knowledge which can be formalized [22,23]. Therefore, we can use that knowledge to manage knowledge in different domain, and then realize the expression and reuse of knowledge.

We define domain individuals, attributes, operations of objects, and the relation between individuals by using Protégé. Protégé is an ontology model developed by 


\section{UI Layer}

\section{Buslness Process Layer}
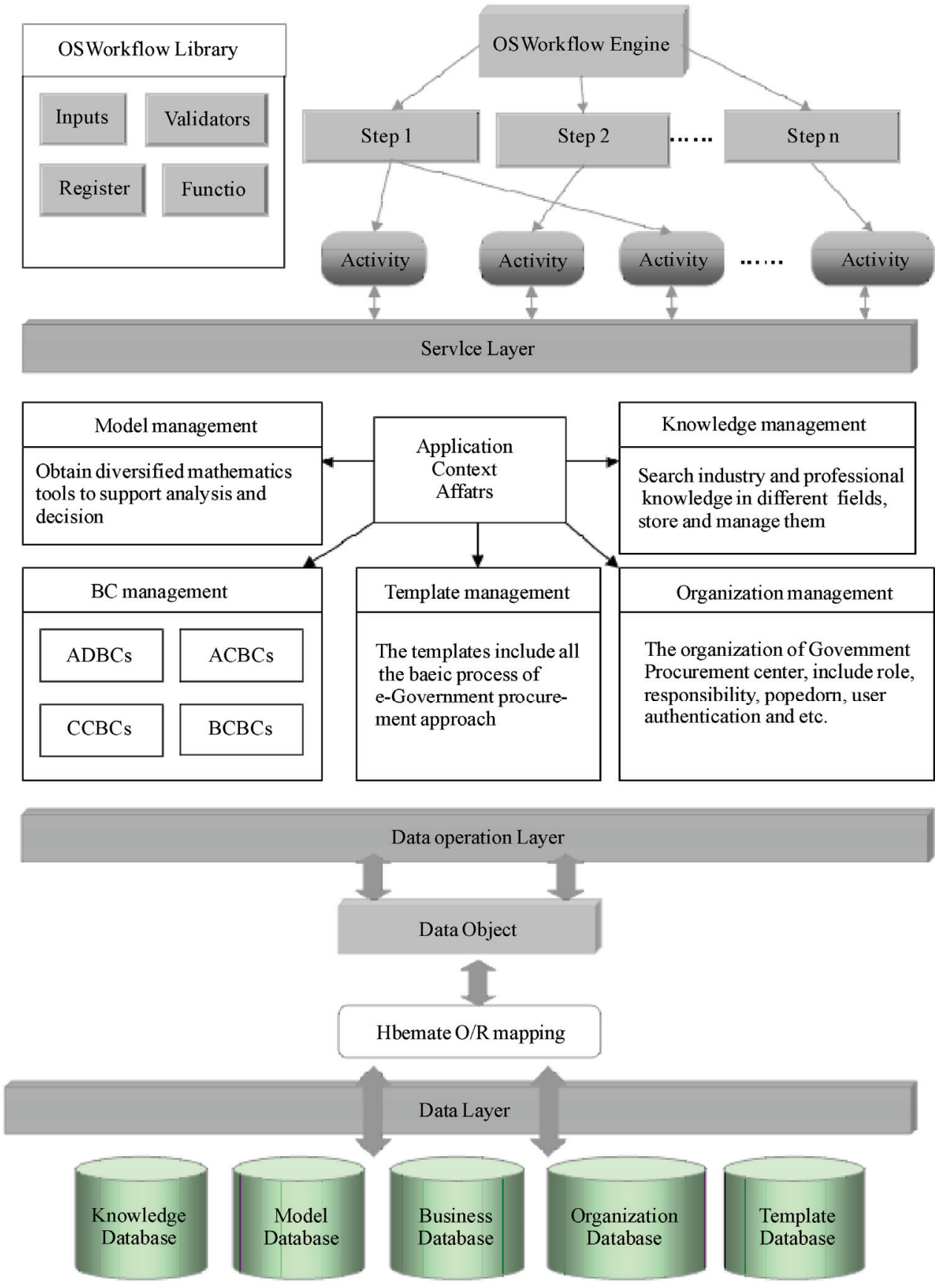

Figure 3. Business component based framework.

Stanford University. It provides a good graphical user interface, and a whole process to compile the figure expression of ontology model, mark and integrate the com- piled file, deploy the model and realize application on it at last. It uses OWL as its ontology language by $\mathrm{OWL}$ Plug-in component. The Jena [24] component provides 
Java API for Protégé.

In this paper, we use Protégé and Osworkflow to do a series of research to realize a prototype of ontology based workflow model.

First of all, ontology of workflow should be described by OWL [23-25]. We take OSworkflow as reference object and design three layers to describe the whole concepts of workflow:

In the first layer, we use OWL to define the concepts of workflow process model and the objects involved, and the Framework of OSworkflow can be seen in Table 1 [20].

In the second layer, we build extended semantic model for workflow ontology to describe the characteristics of each class $[23,25]$. OSworkflow provides a comprehensive grammatical structure for each main class [20]; for example, Activity is a sub-class of workflow process, whose attribute set includes activity number, description, user and roles, information and function resource, preand post-conditions, rollback attribute, etc. We take ADBCs, ACBCs, BCBCs as prototype to define basic activities and sub-processes. Transition has the attribute set of predecessor and successor link, the transfer type and condition. We use OWL to describe those grammatical structures $[22,24]$.

In the third layer, we design domain ontology of government procurement to describe the abstract classes that are expressed by OWL. The abstract classes are mainly used to describe common characteristics of the domain, which can be used as instances to build up Workflow $[26,27]$. The domain can be divided into several classes including Roles, Functions, Projects/Products Tables and so on $[15,28]$. For example, Roles has some common abstract class including suppliers, directors, experts, officials and etc [17]. Different kinds of tables and their characteristics are also described by OWL. The classes contain their basic or common attributes and operations. Detailed information should be provided in the database; for example, we should use ontology to describe the user's

Table 1. Framework of OSworkflow.

\begin{tabular}{lll}
\hline & & Template \\
& & Sub-process(Step) \\
& Process of work- & Activity \\
flow & Connector \\
Concept of Work- & & Transition \\
flow & & Rules \\
\cline { 2 - 3 } & & Information \\
& Organization \\
& Object of workflow & Function \\
& & Resource \\
\hline
\end{tabular}

attributes, the classification of his status, and what kind of operations he should do according to his attributes and status. It is a complicated process.

After building up the ontology of OSworkflow and egovernment procurement, we can export the models from Protégé to MySQL to construct ontology database and thus change ontology-based logical relations into relational database [23].

The main purpose to do that is to build the semantic association between OSworkflow, e-government procurement and their Ontology model. Therefore, two aspects of the work should be finished.

First, translate the ontology model into XML expression which can be implemented by OSworkflow. When we import the elements of ontology database into Protégé, the users can use those elements to define, design and modify the existing workflow model in a graphical interface. After they finish their jobs, the protégé will compile the model, and use RMI to output all the information to a java program $[23,24]$. The java program acts as a translator. First, it identifies the user's role to see if his/her operation is legal, after that, the programs translate the OWL language into XML format and deploy the related file into OSworkflow architecture.

Second, translate the XML format file into OWL language. A typical XML format for OSworkflow to describe the action "apply for procurement" is listed below [20]:

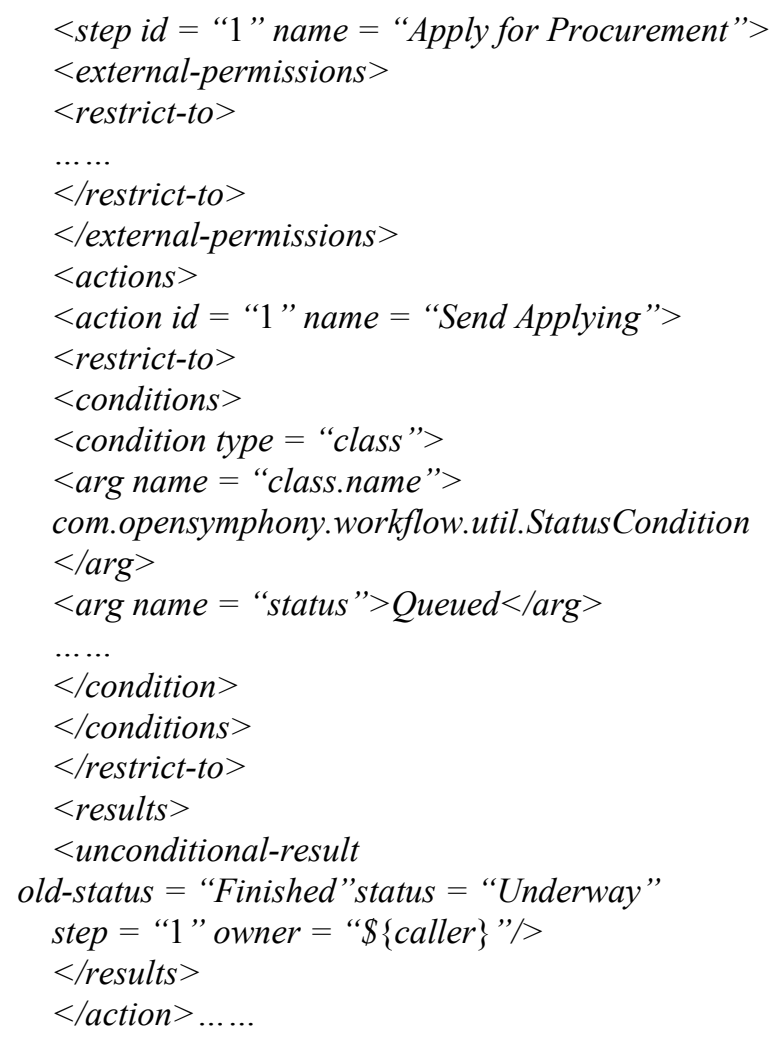


Therefore, to translate the XML is first to find the tags of structure, steps and activities; and then find user, function, condition and others. According to the pre-mapping mechanism, the program can find the related ontology objects in the ontology database to match them, and generate workflow graph by Protégé. The structure of user participation workflow model can be seen in Figure 4.

\section{Instance}

\subsection{Domain Analysis of Public Bidding}

The most representative approach in e-government procurement is public bidding whose steps can be described below:

First, a demand will be produced such as project construction, government service or a large amount of product procurement.

Second, the government procurement center (GPC) whose work is to help government administrators in different hierarchies to implement their procurement plan would make public bidding file (PBF) according to the demand.

Third, the qualified suppliers would quote price and make bidding file (BF) according to the PBF in finite time.

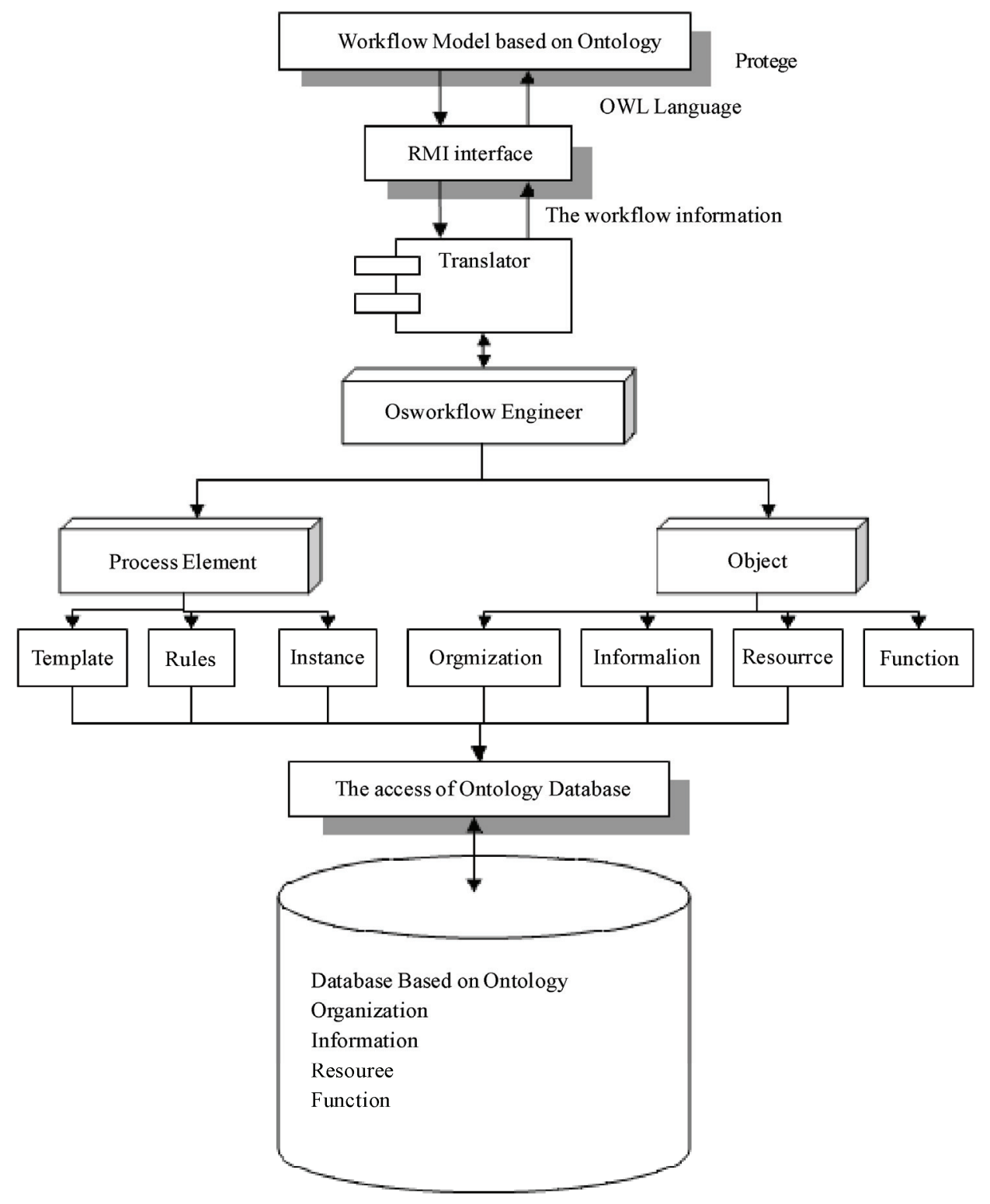

Figure 4. Structure of user participation workflow model. 
Fourth, when the bidding is over, the GPC will invite participants and experts together to hold a conference to decide which company or supplier is the most suitable one to charge of the project or procurement plan.

Based on the domain analysis which is studied from FODA, we summarize the main participants and their activities, divide the activities into meta-process and meta-function, at the same time, use DOA method to build data model, and map the data model into information object such as order table $[18,19]$.

\subsection{Designing BC Framework and BCs}

First, we re-analyze the whole process of public bidding that is executed in twelve different cities in China, abstract common process of public bidding, and compare them with government procurement law in China to find the core processes and functions that can not be modified. Of course, the different parts are also considered as special circumstances to analyze. We use top-down decomposition method to obtain meta-process and metafunction. In order to obtain accurate information to guide the follow work, we consult several university experts and government officials to amend our model.

Second, we divide the model into four parts: decision part, implementation part, supervision part and finance part according to the analysis of their functions and attributes in Section 2. We can design different BCs in different parts. In this paper, we take implementation part as an example to illustrate how to design BCs for government procurement in China.

ADBCs should be designed first, which is the basic procurement process of it. Since it is a process component, it can be divided into many children processes such as applying (P1), auditing application (P2), auditing the authority of suppliers (P3), inviting public bidding (P4), biding (P5), holding the opening bidding meeting (P6), evaluating and publicizing (P7). Each children process can be described as the combination of several metaprocess. Furthermore, each meta-process is required by law and has strict regulation of the process. But they are just framework, the concrete realization of process and function should need other BCs' participation.

The paper uses UML activity diagram to describe the business process of public bidding [28], and translates the diagram into XML format that can be identified by Osworkflow.

When we design $\mathrm{BCs}$, we meet the problem that how to confirm the granularity of BCs. In this paper, we learn from reference [7] to build an index system and use entity-append mechanism to evaluate the performance of the $\mathrm{BC}$ when adding or reducing a meta-process or meta-function on it. By that method, we can optimize the structure of BCs which are specified by clustering algorithm.

ACBCs can be reused by inherit mechanism, since any new instance of $\mathrm{BC}$ can reuse the attributes and functions of ACBCs by extending it. We list representative ACBCs below:

\section{The CRUD of Quotation:}

All e-government procurement approaches should have the CRUD operations on quotations, and there are high similarity in the content of all quotations in different procurement approaches, such as price, type, amount and date. The quotation can be shown in many different types, so we use crystal report forms to design an abstract class which contains several different templates of table format that involve all the main government procurement approaches. We can select different types of tables by configuring the parameters in the abstract class. The structure of Quotation BC can be seen in Figure 5.

We use Osworkflow to create the model of ADBCs, which uses WorkflowNum [20] to specify serial numbers for each process components and the status management mechanism and trigger mechanism to realize the control of all process. The process can be written in XML format in OSworkflow.

\subsection{Integration of Business Component}

The design of ACBCs and BCBCs is supported by Spring [19] architecture. First, set unscramble and management tools of configuration file, then initialize ApplicationContext, whose function is to invoke relevant $\mathrm{BC}$ according to the information which ServiceBeanFactory supplies. We should also define the event mechanism of the whole architecture that can provide standard such as commit, roll back, throw exception and so on for all the activities. Use IoC to define $\mathrm{BC}$ in the configuration file, including the definition, operations, attributes, interface of $\mathrm{BC}$ and the Data transmit object $[18,19]$ (DTO) is one of the most important tools in $\mathrm{BC}$ architecture, it maps the data into data object in XML format, and transmit them to related BCs which need to handle them.

It can be used in different protocol, language and platform which is suitable for system integration. DTO can be designed by reflection mechanism [18,19], and use public package BeanUtil of apache to transfer data object to java object.

ACBCs encapsulate the common functions or processes that can be reused by all the e-government procurement approach in the domain. Therefore the paper adopts Aspect Oriented Programming (AOP) [16,28] technology and Inherit mechanism to allow other BCs use these ACBCs. The most distinct advantage is that it can reduce coupling degree of the system. If we want to 


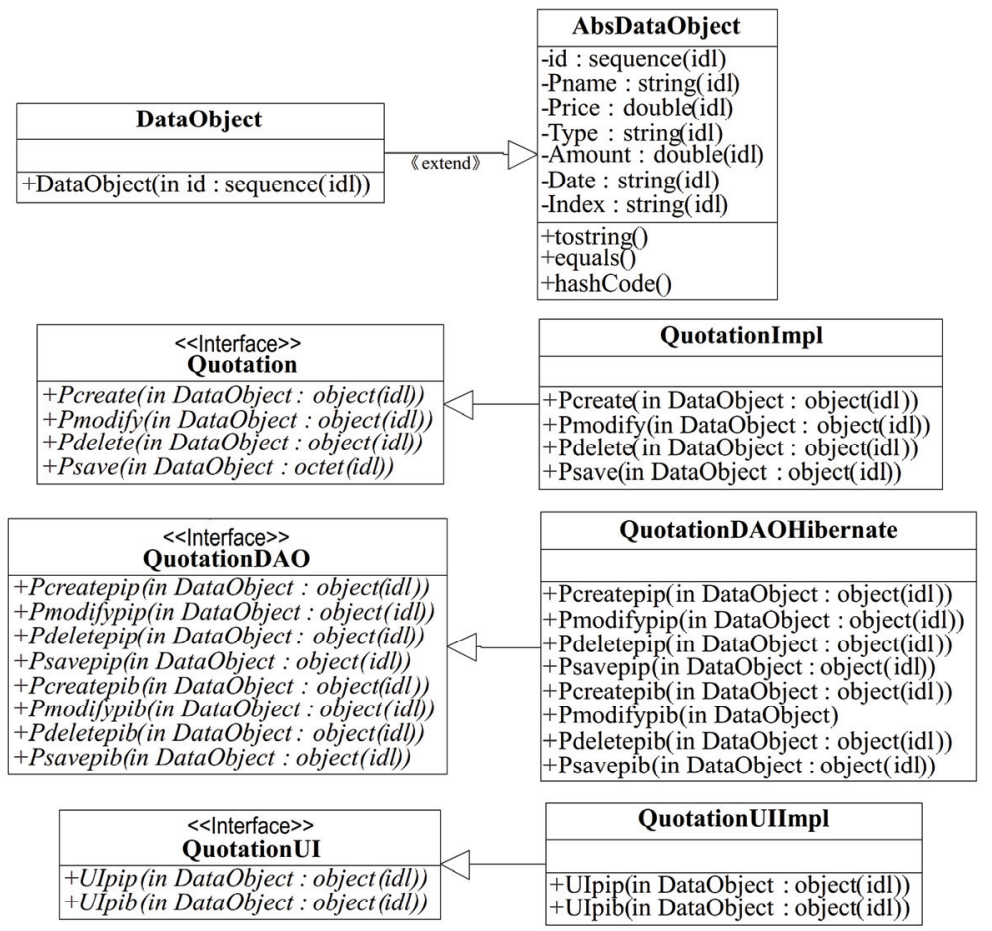

Figure 5. Structure of quotation BC.

modify some common function that is applied in many places of the system, we just need to modify the related $\mathrm{ACBC}$ and deploy it and then the modification will be identified and implemented by the whole system.

\subsection{Evaluation of System Based on BC Methodology}

The system is developed based on an existing open tender system. We redesign the structure of system under the guidance of our framework, decompose the code fragment, and re-integrate them to produce different kinds of BCs, which realize the reuse of existing system.

What's more, ontology is also introduced to design user participation workflow. We mainly make comparisons between the existing system and the new system which is developed under the guidance of our BC framework.

Considering the possible visiting amount of the system, we set the simulative peak visiting amount as 1000 times per minutes, and utilize Equation (1) to calculate the res pond speed of the server under different visiting amount $[22,26,27]$

$$
T=\frac{1}{P}
$$

$T$ : The minimal time unit

\section{$P$ : CPU main frequency}

The contents of system test include: the data amount that is stored in the database during the required time (I1), the jump speed between two different processes (I2), the transfer speed of attachment (I3), the refresh speed of the web pages (I4). After hundreds of tests, we find that when the rate of visiting amount and peak visiting amount reaches $(P V A) 35 \%$, the speed rate of each index can keep a high value compared with ideal speed. When the rate of visiting amount and peak visiting amount reaches $100 \%$, the speed rate of each index can also keep a good value. The data can be seen in Table 2.

Besides, we introduce another two indicators to evaluate the complexity and maturity of the system [22]. The complexity formula can be seen in Equation (2) and the maturity formula can be seen in Equation (3).

$$
\begin{gathered}
C=\frac{N_{r-r}+N_{r-i}+N_{u-u}}{100 \times\left(N_{r}+N_{u}\right)} \\
S=\frac{M_{u}-\left(M_{c}+M_{a}+M_{d}\right)}{M_{p}}
\end{gathered}
$$

$N_{r}$ means the number of classes in the system; $N_{u}$ means the number of web pages in the system; $N_{r-r}$ means the number of relations among different classes, the relationships include inherit and instance; $N_{r-i}$ 
Table 2. System test results.

\begin{tabular}{lllll}
\hline$P V A$ & $I 1$ & $I 2$ & $I 3$ & $I 4$ \\
\hline $35 \%$ & 4200 & $98 \%$ & $85 \%$ & $90 \%$ \\
$100 \%$ & 5200 & $91 \%$ & $71 \%$ & $80 \%$ \\
\hline
\end{tabular}

means the number of the instance relations between class files and web pages. $N_{u-u}$ means the internal relationships among Web pages.

$M_{p}$ means the final numbers of units that is published and deployed. $M_{c}$ means the number of new units compared with original design. $M_{a}$ means the number of the combined or deleted units compared with original design. $M_{d}$ means the number that is changed or modified compared with original design.

According to [22], we know that if the value of $S$ is larger, the system is more mature; if the value of $C$ is smaller, the system is less complexity. The value of $C$ in public bidding system is $43.38 \%$ and $S$ is $73.69 \%$. Compared with the referred existing system, whose value is around $50 \%$, the public bidding system based on $\mathrm{BC}$ framework is more superior.

\section{Conclusions}

First, the paper introduces the development of e-government procurement in China and summarizes both the advantages and deficiencies in recent period. Considering the particulars of e-government, the paper introduces business component theory, and constructs an e-government procurement system framework based on business component. The framework emphasizes process control and provides approach to identify process business components. What's more, we utilize user-participation workflow model based on ontology to realize the flexible design, use Protégé to build ontology model for OSworkflow and e-government procurement, establish semantic association between them and their ontology model.

Meanwhile, the paper also illustrates center management mechanism to manage all the BCs. In order to make the framework more flexible and easy to develop, the framework is divided into 5 layers. Each layer has its BCs. To enhance the reusability of system, the paper introduced 6 different kinds of BCs, and each BC is suit for a particular domain. J2EE light weight development tool is introduced to design the technology architecture of business component framework. At last, the paper takes public bidding as an example to describe how to construct a real system by the knowledge introduced above.

An evaluation test is also exhibited to observe the efficiency of system. The test indicates that enterprise system which is developed by BCs can exhibit more superiority than traditional development method. It is more suitable to be applied in a given domain. The paper also constructs a primary domain BCs library based on ontology for $\mathrm{BC}$ management. By those $\mathrm{BCs}$, it realizes the automatic management of the procurement and provides a higher security mechanism to ensure the impartiality, publicity and equity of e-government procurement.

\section{Acknowledgements}

This research is supported by China National Natural Science Foundation (70971083) and SHUFE Graduate Innovation Foundation (CXJJ-2008-330), Leading Academic Discipline Program, 211 Project for SHUFE (the 3rd phase, 211-5-1), Doctoral Foundation of Ministry of Education (20090078110001).

\section{REFERENCES}

[1] Z. Qin and D. F Li, "The Application Research of EGovernment Procurement in China Based on Business Component Framework," 3rd International Conference on the Digital Society, Cancun, 2009, pp. 30-39.

[2] S. Palkovits and M. Wimmer, "Processes in E-Government - A Holistic Framework for Modelling Electronic Public Services," Electronic Government, 2003, pp. 213219.

[3] China Government Procurement Center. http://www.ccgp. gov.cn/new/

[4] "The Study of Government Procurement in 2008," 2009. http://www.chinanews.com.cn/cj/gncj/news/2009/04-29/1 669788.shtml

[5] "The Situation and the Problem of E-Government Procurement in China," 2006. http://www.china.com.cn/info/ zhuanti/zwfzbg/txt/2006-07/27/content_7028180.htm

[6] W. Kozaczynski, "Architecture Framework for Business Components," 5th International Conference on Software Reuse, Victoria, Computer Society Press, 1998, pp. 300307.

[7] P. Herzum and O. Sims, "Business Components Factory: A Comprehensive Overview of Component-Based Development for the Enterprise," John Wiley, New York, 2000.

[8] C. Szyperski, D. Gruntz and S. Murer, "Component Software: Beyond Object-Oriented Programming," 2nd Edition, Pearson Education Limited, 2003.

[9] Y. Guo, G. Zhang, L. Xie and Y. Xu, "The Research and Design of Business Component Reuse in Enterprise Information System Integration," Proceedings of the 3rd IEEE International Conference of Information Technology and Applications, Sydney, Vol. 2, 2005, pp. 41-44.

[10] H. Jain, N. Chalimeda, N. Ivaturi and B. Reddy, "Business Component Identification: A Formal Approach," The 5th IEEE International Enterprise Distributed Object Computing Conference, Seattle, IEEE Computer Society Press, 2001, pp. 183-187.

[11] J. K. Lee, S. J. Jung, S. D. Kim, W. H. Jang and D. H. Ham, "Component Identification Method with Coupling 
and Cohesion," The 8th Asia-Pacific Software Engineering Conference, Macau, IEEE Computer Society Press, 2001, pp. 79-88.

[12] M. F. Chao, Z. D. Chen and X. X. Fei, "Business Component Identification of Enterprise Information System: A Hierarchical Clustering Method," IEEE International Conference on E-Business Engineering, Beijing, 2005, pp. 1-8.

[13] J. Ginbayashi, R. Yamamoto and K. Hashimoto, "Business Component Framework and Modeling Method for Component-Based Application Architecture," The 4th International Enterprise Distributed Object Computing Conference, Makuhari, 2000, pp. 184-193.

[14] J.-E. Cha, Y.-J. Yang and M.-S. Song, "Design and Implementation of Component Repository for Supporting the Component Based Development Process," IEEE International Conference on Systems, Man, and Cybernetics, Tucson, Washington IEEE Computer Society, 2001, pp. 735-740.

[15] B. Shishkov and J. L. G. Dietz, "Design of Software Applications Using Generic Business Components," Proceedings of the 37th Hawaii International Conference on System Sciences, Big Island, Hawaii, Vol. 9, 2004, pp. 90272b.

[16] B. Eckel, "Thinking in Java," China Machine Press, 2004, pp. 241-256.

[17] H. W. Sui, H. Y. Wang, H. Liu and R. X. Wang, "Design and Implementation of User Permission Management Component System Based on Software Reuse," Computer Application, Vol. 25, No. 5, 2005, pp. 139-143.

[18] L. Zhang, H. Mei and H. Zhu, "A Configuration Management System Supporting Component-Based Software
Development," Computer Software and Applications Conference, IEEE Computer Society, Washington, 2001, pp. 25-30.

[19] R. Harrop, "Guide of Spring Professional Development," Electronic Industrial Publishing Company, Vol. 8, 2006, pp. 125-167.

[20] "Osworkflow". http://www.opensymphony.com/oswor-kflow/

[21] “Appfuse". http://appfuse.org/display/APF/Home

[22] A. Albani and J. L. G. Dietz, "Identifying Business Components on the Basis of an Enterprise Ontology," 1st International Conference on Interoperability of Enterprise Software and Applications, Geneva, 2005, pp. 1-12.

[23] T. B. Lee, J. Hendler and O. Lassila, "The Semantic Web," Scientific American, 2001.

[24] "Jena-A Semantic Web Framework for Java". http://jena. sourceforge.net/

[25] C. Bizer and D. Maynard, "Semantic Web Challenge," 2010. http://challenge.semanticweb.org/

[26] L. Y. Li and P. Li, "The Information System Engineering Supervisor Manual," Publishing House of Electronics Industry, Beijing, 2006, pp. 433-435.

[27] C. C. Zhang, H. X. Xue and P. Tao, "Research on Business Component Modeling in Enterprise Management Application Systems," IEEE International Conference on Management of Innovation and Technology, Singapore, 2006, pp. 916-920.

[28] S. D. Lee and E. S. Cho, "COMO: A UML-Based Component Development Methodology," IEEE International Conference on E-Business Engineering, Los Angeles, 1999, pp. 54-61. 